Ein paar Stunden nach der Narkose erwacht das Schwein und kann bald aufstehen. Zum SchluB zeigen wir Ihnen ein Schwein, das mit einer 12 Std konservierten Leber mehr als 8 Wochen überlebte, ohne in der postoperativen Phase mit Immunosuppressiva behandelt worden zu sein. 3 Tage nach der Transplantation hatten sich die biochemischen Leberwerte normalisiert.

Durch die technisch wenig aufwendige Hypothermie mit intermittierender Perfusion ist es unserer Meinung nach möglich, eine Spenderleber für den klinischen Gebrauch über mehrere Stunden zuverlässig zu konservieren. Das dazu notwendige System läßt sich leicht transportieren.

Wir danken dem Landesforschungsamt Nordrhein-Westfalen und der Deutschen Forschungsgemeinschaft für die gewährte Unterstützung.

\title{
11. Die orthotope Lebertransplantation beim Hund
}

\section{Farbfilm}

\section{Lempinen, K. SAlmenkivi und A. Sivula-Helsinki/Finnland}

In dem Film werden die allgemeinen Richtlinien der Lebertransplantation und die folgenden drei Phasen der operativen Prozedur dargestellt:

1. Die Präparation des Spenderhundes,

2. Die Präparation des Empfängerhundes una

3. Die Rekonstruktionsphase mit den erforderlichen fünf Anastomosen.

Dazu werden die wichtigsten Prinzipien und Einzelheiten mit schematischen Zeichnungen dargestellt.

Der Spender wird in einer Hypothermie von $30^{\circ} \mathrm{C}$ hepatektomiert. Zuerst werden die Gefäße der Leber freipräpariert, und die Perfusion der Leber wird bereits in situ mit einer buffertierten und bis zu $4^{\circ} \mathrm{C}$ abgekühlten Hartmanns-Flüssigkeit begonnen. Die Perfusion wird dann nach der Separation der Leber ergänzt und bis zum Beginn der Re-Implantation fortgesetzt.

Der Empfänger wird normothermisch laparotomiert und hepatektomiert. Die hämodynamischen Arrangements umfassen sowohl ein porto-jugulares als ein femoro-jugulares Shunt-Verfahren mit einem extrakorporalen Schlauch ohne motorische Pumpe.

In der Rekonstruktionsphase werden folgende Anastomosen in erwähnter Reihenfolge genäht: End).

1. Die suprahepatische Hohlvenenanastomose unter dem Zwerchfell (End-zu-

2. Die Pfortaderanastomose (End-zu-End), so daß der größte Teil der Cireumferenz genäht wird, solange noch ein Spezialkatheter im Stumpf der EmpfängerPfortader daliegt.

3. Die infrahepatische Hohlvenenanastomose (End-zu-End).

4. Die Arterienanastomose (End-zu-End) zwischen dem Zöliaka-Stumpf des Spenders und dem Renalis-Stumpf des Empfängers, dessen rechte Niere weggenommen worden ist.

5. Eine Cholecystojejunostomie mit einer Y-Anastomose nach Roux. Zuletzt wird das postoperative Verhalten von zwei Hunden bis zu zwei Monaten bzw. drei Wochen nach der Transplantation geschildert. Beide Hunde bekamen Azathioprin (Imurel@) und Prednison als immunosuppressive Therapie. 